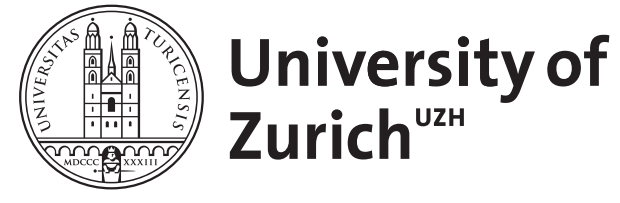

Zurich Open Repository and Archive

University of Zurich

University Library

Strickhofstrasse 39

CH-8057 Zurich

www.zora.uzh.ch

Year: 1987

\title{
Zur Frage nach der Begründung des Naturschutzes
}

\author{
Ruh, Hans
}

DOI: https://doi.org/10.14315/zee-1987-0113

Posted at the Zurich Open Repository and Archive, University of Zurich

ZORA URL: https://doi.org/10.5167/uzh-154349

Journal Article

Published Version

Originally published at:

Ruh, Hans (1987). Zur Frage nach der Begründung des Naturschutzes. Zeitschrift für evangelische Ethik, $31(1): 125$.

DOI: https://doi.org/10.14315/zee-1987-0113 


\section{Kommentar}

\section{Zur Frage nach der Begründung des Naturschutzes ${ }^{1}$}

\section{VON HANS RUH}

Einerseits ist Naturschutz ein verbreitetes Thema geworden. Andererseits sieht sich mancher in nicht geringer Verlegenheit, wenn er triftige Argumente für den Schutz der Natur formulieren muß. Schon die Definition des Begriffs macht Mühe. Zwar hat G. M. Teutsch im Lexikon der Umweltethik ${ }^{2}$ eine brauchbare Definition vorgelegt: "Naturschutz ist der umfassende Begriff für alle Bestrebungen und Maßnahmen, natürliche oder naturnahe Regionen vor weiterer Erschließung, Nutzbarmachung oder Überbauung zu schützen, um diese Landschaften als solche, aber auch als Lebensräume für gefährdete Tier- und Pflanzenarten oder zur Erhaltung überkommener Naturdenkmale zu schützen. Obwohl der Naturschutz schon im letzten Jahrhundert begann, wird er heute als Teil des ganz jungen Umweltschutzes betrachtet. So verliert er seine eigene ethische Begründung und gerät immer mehr in den Sog der im Umweltschutz vorherrschenden anthropozentrischen Umweltethik.«

Trotzdem muß man weiter fragen: Was und wieviel von was soll denn nun geschützt werden? Auf welcher Ebene bewegt sich Naturschutz in Abgrenzung zu Tier- und Pflanzenschutz? Geht es um den Schutz von Zellen, Individuen, Populationen, Arten, Lebensgemeinschaften, Lebensräumen, Lebensgrundlagen? Auch wenn also zur Definition noch nicht das letzte Wort gesagt ist, setzen wir im folgenden die nach Teutsch zitierte voraus, weil es uns zentral und ausschließlich um die Begründung geht.

Die gesetzlichen Artikel, welche sich mit dem Naturschutz befassen, sind weit herum ausschließlich anthropozentrisch formuliert. Aber auch diese begrenzte Begründung bedarf der Rechtfertigung. Sie kann mit der folgenden Norm erfolgen: Alle 
beute aktuell und in Zukunft potentiell lebenden Menschen haben das gleiche Recht auf Leben und Entfaltung, es sei denn, es gäbe triftige ökologischbiologische Gründe, die dagegen sprächen. Zur Begründung dieser Norm lassen sich folgende Überlegungen anführen: Menschen, die gerne leben und alles vorkehren, um das Leben zu schützen, zu verlängern, zu verbessern, können nicht gleichzeitig sagen, daß die Frage, ob zukünftige Menschen auch leben sollen, egal oder unentscheidbar sei. Aus ethischen und zugleich logischen Gründen kann die Gemeinschaft der gerne leben wollenden Menschen nur das Recht auf Weiterleben der Menschheit anerkennen. (Allenfalls hätte eine Gemeinschaft von zum Selbstmord entschlossenen Menschen das Recht, diese Frage anders zu beantworten.)

Von daher ergibt sich der Schluß, daß die Natur zumindest als Voraussetzung für die Lebensmöglichkeit der menschlichen Gattung geschützt werden soll, denn der Mensch kann ohne (übrige) Natur nicht existieren. Zwar gibt es Theorien, wonach der Mensch in einer vollständig synthetisierten Umgebung weiterleben könne. Die ökologisch-biologischen Gründe gegen solche Theorien sind aber einleuchtend; ganz abgesehen davon ist aus ethischer (und ökonomisch-sozialer) Sicht nicht zu begründen, daß im Weiterleben in einer vollständig synthetisierten Umgebung irgendein Vorteil zu erblicken sein soll.

Die wirklich interessante Frage ist aber doch wohl, ob es Gründe für den Naturschutz außerhalb des menschlichen Interessenbereiches gibt. Sucht man unter diesem Aspekt nach Argumenten, dann wird man plötzlich gewahr, daß in der oben entfalteten Norm, welche sich auf das Leben der Menschen bezieht, noch mehr steckt.

Faßt man den Grundsatz, wonach jeder Mensch das gleiche Recht auf Leben hat, prinzipiell und radikal, so wird bald deutlich, daß es keine Gründe gibt, dieses Recht auf Leben nicht auf alles Leben auszudehnen. Denn letztlich haben wir das Recht der zukünftigen Menschen auf Leben gefolgert aus dem Willen der heutigen Menschen zum Leben. Diesen Willen zum Leben (A. Schweitzer) müssen wir aber bei allem Lebendigen in gleicher oder vergleichbarer Weise voraussetzen. Damit ergibt sich die Folgerung, daß wir den ersten Grundsatz auf alles Leben erweitern 
müssen. Er lautet nun: Jedes Lebewesen hat das gleiche Recht auf Leben.

Mit diesen Feststellungen stoßen wir vor zu einer grundsätzlichen, den anthropozentrischen Ansatz überholenden Begründung des Naturschutzes: Weil alle Lebewesen das gleiche Recht auf gegenwärtiges und zukünftiges Leben haben, müssen prinzipiell alle Lebewesen und ihre Lebensmöglichkeiten in gleicher Weise geschützt werden. (Dies gilt natürlich vorerst alles "nur» aus ethischer Sicht.)

Geht man nun aus von den bisher gerechtfertigten zwei Normen, sind einige Begründungen für den Naturschutz möglich, natürlich unter Berücksichtigung einiger anderer Normen, die wir auch als gerechtfertigt ansehen können, so etwa:

- Jedermann hat Anspruch auf gerechte Verteilung unter Berücksichtigung seiner Leistung und seiner Bedürfnisse.

- Jedermann hat die Pflicht, für sich und für die Allgemeinheit einen angemessenen Beitrag zur Bewältigung der Lebensprobleme zu leisten.

- Gleiches ist gleich zu behandeln.

- Unverschuldete Ungleichheit ist auszugleichen.

Ausgehend von den bisherigen Überlegungen soll nun versucht werden, ethische Argumente für den Naturschutz zu finden. Gesucht werden Argumente, denen man nicht widersprechen kann, wenn man

- den oben beschriebenen ethischen Standort akzeptiert und

- bereit ist, in fairer Weise im Rahmen der Logik zu argumentieren.

Argumente für den Naturschutz in diesem Sinne werden im folgenden auf verschiedenen Ebenen gesucht. Zunächst folgt eine Übersicht über die Systematik der Ebenen bzw. der unterschiedlichen Zugänge zu einer ethischen Argumentation:

- Aufgeklärtes Eigeninteresse des Menschen,

- gerechte Verteilung unter den heute lebenden Menschen,

- Rechte der zukünftigen Generationen,

- Rechte der Natur, die aus der vom Menschen erkannten Analogie des Menschen zur übrigen Natur gefolgert werden,

- Eigenrechte der Natur aufgrund von in der Natur entdeckten Ordnungsprinzipien, 
- ethische Folgerungen aus dem Nicht-Wissen des Menschen über die Natur,

- aus dem Können des Menschen abzuleitende Pflichten gegenüber der Natur,

- aus den Eingriffen des Menschen in die Natur abgeleitete Pflichten,

- Ehrfurcht vor der Würde des in langer Zeit Gewordenen.

\section{Aufgeklärtes Eigeninteresse des Menschen.}

Der Mensch kann nicht ohne die Natur existieren. Biologische, medizinische, ökonomische und psychologische Ansprüche entsprechen überlebensnotwendigen Bedürfnissen des Menschen. Es gibt also ein wohlverstandenes Eigeninteresse des Menschen am Schutz der Natur, wie es etwa in gewissen Naturschutzgesetzen formuliert wird.

Insofern die Ethik die aufgeklärten Eigeninteressen des Menschen positiv bewertet ( $z$. B. durch den Verweis auf das Recht auf Leben), ist diese erste Argumentationsebene auch ethisch relevant.

\section{Gerechte Verteilung unter den beute lebenden Menschen.}

Wir erleben heute, daß Menschen durch das Verhalten anderer Menschen gleich mehrfach benachteiligt werden: Wer aufgrund seiner ökonomischen Möglichkeiten einen Lebensstil hat, der die Natur stark belastet, schafft z. B. Umweltprobleme auch für die Menschen, welche bescheiden leben (müssen) und/oder die Natur wenig belasten. Naturschutz ist deshalb anzustreben zum Zweck der Erhaltung der Natur für alle. Ethisch problematisch ist auf jeden Fall eine Zerstörung der Natur bzw. die Schaffung von Umweltbelastungen, welche den Durchschnitt der Belastung pro einzelnes Individuum überschreiten.

\section{Rechte der zukünftigen Generationen.}

Vom Grundsatz ausgehend, daß alle, auch die zukünftigen Menschen, das gleiche Recht auf Leben und Entfaltung haben, ist die Forderung abzuleiten, daß die Wahrung des Zuganges zur Natur bzw. zu den Gütern der Natur prinzipiell auf einem Niveau zu halten ist, das den zukünftigen Generationen Gleichheit garantiert. Natürlich wird man dabei das unterschiedliche zivilisato- 
risch-technologische Niveau angemessen berücksichtigen, aber auch die Bevölkerungsentwicklung. Auf alle Fälle muß derjenige Grundbestand der Natur gewahrt bleiben, der es unter allen Umständen zukünftigen Generationen erlaubt, ihre Rechte wahrzunehmen. Ein Beispiel ist etwa der Umstand, daß die Natur eine Vielzahl von heute in ihrer Tragweite noch nicht erkannten natürlichen Stoffen bereithält, welche später lebenswichtig sein könnten. Zum Grundbestand der Natur gehört die Erhaltung etwa klimatischer Rahmenbedingungen, der Bodenschätze, der Wasser- und Bodenqualität etc.

An dieser Frage wird deutlich, daß das im Anschluß an Max Weber formulierte Prinzip, wonach der Mensch nur für diejenigen Folgen verantwortlich zu machen sei, die vorauszusehen sind, seine Gültigkeit eingebüßt hat. Es ist vielmehr geradezu umzukehren: Der Mensch darf sich, in bezug auf die tiefgreifenden Einwirkungen auf die Natur, nur auf Strategien einlassen, von denen er weiß, daß die Folgen vor dem Prinzip gleichen Rechtes zukünftiger Generationen bestehen können.

4. Recbte der Natur, die aus der vom Menschen erkannten Analogie des Menschen zur übrigen Natur gefolgert werden.

Ausgangspunkt der Überlegungen auf dieser Ebene ist zunächst die schon getroffene Feststellung, daß der Mensch leben will und daß er damit das gleiche Recht auf Leben für die anderen Menschen anerkennen muß. Hier ist aber zu erinnern an eine weitere grundlegende Norm, wonach Gleiches gleich zu behandeln sei. Nun haben wir einleitend keinen Zweifel darüber gelassen, daß alle Lebewesen auf Leben aus sind. D. h., man kann bei allen Lebewesen, nach der Definition von Albert Schweitzer, den Willen zum Leben voraussetzen. Es gibt aber, auf dieser grundsätzlichen Ebene, keinen Grund, von diesem allgemeinen Willen zum Leben aus nicht ein allgemeines, dem menschlichen vergleichbares Recht auf Leben abzuleiten. Und so haben wir den Grundsatz: JJeder Mensch hat das gleiche Recht auf Leben` zu dem Satz: >Jedes lebendige Wesen hat das gleiche Recht auf Leben، erweitert. Dieser Grundsatz scheint sich nur auf Individuen zu beziehen. In seiner Konsequenz bezieht er sich aber auch auf Arten von Lebewesen; denn zum Recht auf Leben (und Entfaltung) 
gehört doch wohl beim Menschen wie bei anderen Lebewesen, daß sie sich fortpflanzen können. Damit wird hier nicht weniger als eine ethische Begründung für den generellen Artenschutz behauptet. Ausgeklammert wird hier vorläufig das Problem, daß es unweigerlich zum Konflikt zwischen den verschiedensten Ansprüchen (und Rechten) auf Leben kommt. Diese Konfliktregelung muß aber auf einer anderen Ebene angegangen werden. Wir sagen hier nur soviel: Alle Lebewesen haben das gleiche Recht auf Leben und Entfaltung; dieses Recht kann durch Erfordernisse der Konfliktbewältigung eingeschränkt werden. Es kann auch durch ökologische Erfordernisse eingeschränkt werden, sofern triftige ökologisch-biologische Gründe dagegen sprechen.

\section{Eigenrechte der Natur aufgrund von in der Natur qu entdeckenden Ordnungsprinzipien der Natur.}

Hier bewegen wir uns insofern auf erkenntnistheoretisch schwierigem Gelände, als es strikte gesehen keine nicht-anthropomorphen Aussagen über die Natur gibt. Trotzdem muß es erlaubt sein, den Versuch, von der Natur her zu denken, zu machen. Gibt es also Eigenrechte der Natur, unabhängig von den Bedürfnissen und der ethischen Logik des Menschen? Die Beantwortung dieser Frage ist nicht leicht, denn die Natur ist in allen ihren Teilen Leben; und es ist zunächst nicht auszumachen, welche Teile mehr Rechte haben sollen als andere. So ist zunächst, auf dieser Ebene, nicht auszumachen, ob der Borkenkäfer oder die Tanne, das Kraut oder das Unkraut, überleben sollen. Aber heißt das, daß auf jede ökologisch-biologische Begründung des Naturschutzes verzichtet werden muß?

Es könnte weiterführen, hier einmal die Frage nach dem Ziel der Natur zu stellen: Offenbar will die Natur eine Vielfalt anstreben; sie will die ständige Vermusterung, und sie strebt nach einer immer höheren Stufe der Evolution. Nun kann man einwenden, es sei der Natur gleich, wenn sie immer wieder von vorne anfangen müsse. Trotzdem ist nicht einzusehen, warum wir nicht das zu respektieren haben, was wir als Ziel der Natur erkennen können. Zugleich können wir in dem Ziel der Vermusterung auch einen ökologischen Sinn erkennen, nämlich den der Stabilität des Systems. 
Prominent wird diese Position von C. Bresch, Zwischenstufe leben, Evolution ohne Ziel? vertreten. Bresch argumentiert etwa so: Wir müssen so handeln, daß die Folgen unseres Handelns nicht mit dem Ziel der Natur in Konflikt geraten. Das Ziel der Natur ist die wachsende Vermusterung. "Evolution ist - zumindest bis heute und zumindest im Erfahrungsbereich aller menschlichen Wissenschaft - ein unaufhörlich beschleunigtes Wachstum von Mustern. Evolution hat also eine erkennbare, stets gleichbleibende Richtung: Sie läuft zu immer weiter vernetzten und komplexeren Zuständen der Materie dieser Welt « ${ }^{3}$. Aus der erkannten Richtung, in die sich die Natur, manifestiert in der "Tatsache« der Evolution, entwickelt, kann man auch ein Ziel der Natur erkennen, dann nämlich, wenn die eingeschlagene Richtung zu ständiger Vergrößerung der Komplexität zwingend ist im Sinne einer inhärenten Systemeigenschaft. Wenn wir aber das Ziel der Natur erkennen können, müssen wir ihr auch das Recht zugestehen, es zu verfolgen, und wir dürfen keine Maßnahmen treffen, welche mit diesem Recht unvereinbar sind, es sei denn, es gäbe Rechte des Menschen, welche höher einzustufen wären, oder es gäbe triftige ökologische Gründe dafür.

\section{Etbische Folgerungen aus dem Nicht-Wissen des Menschen über die Natur.}

Nicht bloß vom Wissen, auch vom Nicht-Wissen, lassen sich ethische Folgerungen ableiten. Gerade wer einwendet, daß die Beurteilung des Zieles der Natur, also die Vermusterung, auf wackligem Boden stehe, muß gleichzeitig anerkennen, daß auch das Nicht-Wissen ethisch bedeutungsvoll sein kann. So wissen wir nicht, ob bei einer gravierenden Zerstörung der Natur, z. B. durch Umweltkatastrophen oder Atomkrieg, die Natur je wieder in der Lage sein wird, den gegenwärtigen Stand der Evolution zu erreichen. $\mathrm{Da} ß$ sie ihn erreichen will, hat sie gezeigt. Ob sie ihn wieder erreicht, wenn wir durch einen Atomkrieg 2 Milliarden Jahre Evolution rückgängig machen, ist schon durch den Hinweis auf das mögliche Versiegen von Sonnenenergie in 2 Milliarden Jahren fraglich. Es ist dies aber auch sonst fraglich, denn wie sollten sich die allenfalls vorauszusetzenden "Zufälligkeiten" wiederholen. Und auch der Hinweis auf weitere 200000 mögliche Sonnensy- 
steme mit Lebensmöglichkeiten hilft nicht viel, weil dies alles Spekulationen sind, insbesondere hinsichtlich anderer Systeme. Wir müssen deshalb sagen: Weil wir nicht wissen, ob sich die Evolution wiederholen läßt, ist Artenschutz begründet.

Allerdings müssen wir hier noch etwas präziser werden. Genaugenommen läßt sich nur soviel eindeutig sagen: Entweder leiten wit den Artenschutz von unserem Analogieschluß (4) bzw. von unserem ökologischen Wissen (5) ab oder aber von unserem Nicht-Wissen (6). Diese Alternative ist gegeben, aber sie ist in dem Sinne zwingend gegeben, daß wir das eine oder das andere wählen müssen.

7. Aus dem Können des Menschen abzuleitende Pflicbten des Menschen. Jeder Mensch hat eine Pflicht gegenüber der Gesamtheit. Diese Pflicht läßt sich, nach den Feststellungen über den Willen zum Leben, auch auf die nichtmenschliche Gemeinschaft ausdehnen. Die ethischen Postulate, die wir bisher entfaltet haben, sind also nicht einfach der Freiwilligkeit anheimgestellt. In dem Maße, in dem der Mensch eingreifen kann im Sinne der Postulate, muß er es tun.

8. Aus den Eingriffen des Menschen in die Natur abgeleitete Pflichten. Der Mensch hat die Natur verändert, indem er massiv in sie eingegriffen hat. Das Sonderverhalten des Menschen zeigt, daß er, wie kein anderes Lebewesen, selbst, bewußt, aufgrund strategischer Konzeption, massiv in die Natur eingegriffen hat. Dies gilt z. B. für die Quantität der Population, die Zerstörung anderer Arten, die Artefaktifizierung (Städte, Straßen, Klima), die Umweltbelastung. Man stelle sich vor, daß andere Arten sich in entsprechender Anzahl und Massierung wie die Menschen benehmen! Dazu kommt noch, daß der Mensch in die Selektion der Natur bewußt eingreift. Er hat damit einen Teil der Selbststeuerung der Natur usurpiert. Damit ist er teilweise für die Folgen selbst verantwortlich und verpflichtet, seine Steuerung zu verantworten und weiterzuführen. Damit ist er aber in die Notwendigkeit versetzt, Grundsätze für die Steuerung aufzustellen und zu beachten.

Auch unter diesem Aspekt ist die Freiwilligkeit ethisch orien- 
tierten Handelns in der Natur aufgehoben: Der Mensch muß handeln, und damit steht er unter dem Zwang, seinem Handeln ethische Grundsätze zu geben. Diese können aber schwerlich andere sein als diejenigen, die wir unter den verschiedenen Punkten hier aufgestellt haben. Sollte dies nicht der Fall sein, so müßte gesagt werden, wie denn andere Grundsätze zu lauten haben.

\section{Ebrfurcht vor der Würde des in langer Zeit Gewordenen.}

Eingriffe des Menschen in die Evolution sind Eingriffe in etwas, das in langer Zeit geworden ist und das der Mensch weder geschaffen hat noch wieder schaffen kann. Es kann nicht plausibel sein, daß ein Teil eines Systems massiv und zerstörend in das System eingreifen darf, wenn er dieses System nicht wieder schaffen noch verbessern kann, ja es überhaupt nicht geschaffen hat. Viel plausibler ist die Annahme, daß ein Teil des Systems die Würde des ganzen Systems zu respektieren hat.

Dieser Versuch der Beibringung von Argumenten für den umfassenden, die menschlichen Interessen überholenden Naturschutz enthält so ethische und ökologische Erwägungen. Natürlich müßte in einem nächsten Schritt die Frage der Konfliktregelung grundsätzlich aufgegriffen werden, denn zwischen Mensch und nichtmenschlicher Natur existieren harte Gegensätze. Dieses Problem zu lösen ist hier nicht intendiert. Unsere Überlegungen beschränken sich allein auf die Frage, worin denn triftige Gründe für den Naturschütz bestehen könnten.

Prof. Dr. Hans Rub Institut für Sozialethik
der Universität Zürich
Kirchgasse 9
CH-8001 Zürich

\section{Anmerkungen}

1. Die folgenden Ausführungen stützen sich teilweise auf Resultate eines Seminars an der Universität Zürich im WS 1986/87 unter dem Titel „Ist Naturschutz ethisch oder ökologisch zu begründen?

2. Göttingen 1985, S. 77.

3. Fischer TB, Frankfurt/M. 1977, S. 269. 